\section{Liver/kidney microsomal antibody type 1 and liver cytosol antibody type 1 concentrations in type 2 autoimmune hepatitis}

\author{
L Muratori, M Cataleta, P Muratori, M Lenzi, F B Bianchi
}

equivocally proven. ${ }^{12}$ As in the case of many other organ specific autoimmune disorders, autoreactivity specific to $\mathrm{AIH}$ and directed against a liver specific component, conceivably expressed on the hepatocyte plasma membrane, was postulated and searched for from the very beginning, but to little avail.

Type 2 AIH represents a serologically and clinically distinct subset of $\mathrm{AIH}$, generally negative for anti-nuclear antibodies and antismooth muscle antibodies, but positive for liver/kidney microsomal antibody type 1 (LKM1) and liver cytosol antibody type 1 (LC1). ${ }^{3-7}$ The main antigenic target of LKM1 in type $2 \mathrm{AIH}$ is cytochrome P450IID6 (CYP2D6), a $50 \mathrm{kDa}$ microsomal protein involved in the metabolism of xenobiotics, ${ }^{8}$ and in particular its sequence spanning amino acid positions $257-269$ is considered to be the most frequently recognised linear epitope. ${ }^{9}{ }^{10}$ The demonstration that CYP2D6 is present and functional on the outer surface of the plasma membrane of human hepatocytes, ${ }^{11}$ although not universally accepted, ${ }^{12}$ supports the hypothesis that LKM1 reactivity, besides its diagnostic value, may also have pathogenic implications in type 2 AIH. $^{13} \mathrm{LC} 1$ is a liver specific autoantibody detectable, either alone or in association with LKM1, in a significant proportion of patients with type $2 \mathrm{AIH} \cdot{ }^{14-17}$ To date, only scarce information is available on its target, the most appealing of all being that LC1 antigen is strictly confined to the liver. ${ }^{7} \mathrm{By}$ indirect immunofluorescence on rat liver sections, LC1 positive sera stain homogeneously the cytoplasmic compartment of periportal, but not perivenular, hepatocytes, indicating that the target antigen is not uniformly distributed in rodent substrates. When LKM1 is also present in the same serum, the distinguishing LC1 immunofluorescence is obscured by the LKM1 pattern, and other techniques such as immunodiffusion and counterimmunoelectrophoresis are required for its identification. In addition, the precipitin line of identity with a positive reference serum obtained by immunodiffusion or counterimmunoelectrophoresis confirms the presence of LC1. ${ }^{714}{ }^{17} \mathrm{By} \mathrm{immu}-$ noblotting, LC1 positive sera recognise a liver specific cytosolic protein of $58-62 \mathrm{kDa}^{14-17}$ Interestingly, it appears that LC1 antigen is particularly well represented in the cytosolic fraction of human liver. ${ }^{15}{ }^{16}$ At variance with the LKM1 autoantigen, at present there are no data indicating that the whole LC1 protein or its processed fragments are exposed on the hepatocyte plasma membrane. Should this be
More than 40 years since its first formal description, autoimmune hepatitis (AIH) is still considered to be a cryptogenic necroinflammatory liver disorder, its pathogenesis has remained largely unknown, and even its autoimmune nature is far from being un- 
Table 1 Clinical, biochemical, immunological, and histological features at presentation in the 21 patients with type 2 autoimmune hepatitis

\begin{tabular}{|c|c|c|c|c|c|c|c|c|c|c|}
\hline Patient & Age (y) & $\operatorname{Sex}$ & Autoantibodies & $\begin{array}{l}\text { ALT ( } \times \\
\text { normal) }\end{array}$ & $\begin{array}{l}\text { AST ( } \times \\
\text { normal) }\end{array}$ & $\begin{array}{l}\text { Albumin } \\
(\mathrm{g} / \mathrm{l})\end{array}$ & $\underset{(g / l)}{\gamma \text { Globulin }}$ & $\operatorname{Ig} G(g / l)$ & Liver histology & IAHG score \\
\hline 1 & 13 & $\mathrm{~F}$ & LC1 & 10 & 7.5 & 28 & 40 & 57 & Severe $\mathrm{CAH}$ & $19 \mathrm{~d}$ \\
\hline 2 & 7 & $\mathrm{~F}$ & LKM1 & 20 & 14 & NA & 30 & 33.2 & Severe CAH & $19 \mathrm{~d}$ \\
\hline 3 & 9 & $\mathrm{~F}$ & LC1 & 15 & 6 & 46 & 14 & 19.2 & Severe CAH & $16 p$ \\
\hline 4 & 25 & $\mathrm{~F}$ & LKM1 & 1.5 & 2 & 19 & 23 & 24 & NA & $17 \mathrm{~d}$ \\
\hline 5 & 10 & $M$ & LKM1/LC1 & 8 & 5 & 42 & 17 & 24.1 & Severe CAH & $15 p$ \\
\hline 6 & 5 & $\mathrm{~F}$ & LC1 & 35 & 30 & NA & NA & NA & Active cirrhosis & $19 \mathrm{~d}$ \\
\hline 7 & 9 & $\mathrm{~F}$ & LKM1 & 4 & 4 & 45 & 36 & 39 & Active cirrhosis & $20 \mathrm{~d}$ \\
\hline 8 & 19 & $\mathrm{~F}$ & LKM1/LC1 & 2 & 1.5 & 30 & 23 & NA & NA & $20 \mathrm{~d}$ \\
\hline 9 & 8 & $\mathrm{~F}$ & LKM1 & 10 & 7 & 31 & 48 & 39.7 & Active cirrhosis & $20 \mathrm{~d}$ \\
\hline 10 & 18 & $\mathrm{~F}$ & LKM1 & 80 & 70 & 43 & 15 & 24.5 & Severe CAH & $18 \mathrm{~d}$ \\
\hline 11 & 20 & $\mathrm{~F}$ & LC1 & 17 & 11 & 40 & 28 & NA & Severe $\mathrm{CAH}$ & $18 \mathrm{~d}$ \\
\hline 12 & 4 & $\mathrm{~F}$ & LC1 & 60 & 70 & 35 & 23 & 31 & Severe CAH & $16 \mathrm{p}$ \\
\hline 13 & 3 & $\mathrm{~F}$ & LKM1/LC1 & 15 & 19 & 29 & 40 & 49.9 & Active cirrhosis & $19 \mathrm{~d}$ \\
\hline 14 & 10 & $M$ & LKM1/LC1 & 3 & 2 & 45 & 19 & NA & Active cirrhosis & $16 \mathrm{p}$ \\
\hline 15 & 24 & $\mathrm{~F}$ & LC1 & 3 & 1 & 28 & 26 & NA & NA & $16 \mathrm{~d}$ \\
\hline 16 & 1 & $\mathrm{~F}$ & LKM1 & 40 & 69 & NA & NA & NA & Severe $\mathrm{CAH}$ & $19 \mathrm{~d}$ \\
\hline 17 & 8 & $\mathrm{~F}$ & LC1 & 35 & 40 & NA & 36 & 24.8 & Severe CAH & $18 \mathrm{~d}$ \\
\hline 18 & 5 & $\mathrm{~F}$ & LC1 & 15 & 20 & 44 & 14 & 21 & Severe CAH & $18 \mathrm{~d}$ \\
\hline 19 & 15 & $M$ & LC1 & 26 & 27 & 34 & 32 & 34.6 & $M N$ & $16 p$ \\
\hline 20 & 14 & $\mathrm{~F}$ & LKM1/LC1 & 20 & NA & 35 & 38 & 50 & Severe CAH & $19 \mathrm{~d}$ \\
\hline 21 & 30 & $\mathrm{~F}$ & LKM1 & 5 & 4 & 40 & 26 & 25.8 & Severe CAH & $18 \mathrm{~d}$ \\
\hline
\end{tabular}

ALT, serum alanine aminotransferase; AST, serum aspartate aminotransferase; LC1, liver cytosol antibody type 1; LKM1, liver/kidney microsomal antibody type 1; NA, not available; CAH, chronic active hepatitis; $\mathrm{MN}$, multilobular necrosis; IAHG, International Autoimmune Hepatitis Group; d, definite autoimmune hepatitis; p, probable autoimmune hepatitis.

demonstrated, the LC1 antigen could well be considered as a potential candidate for liver targeted autoimmune reactions.

The aim of this study was to determine the concentrations of LKM1 and LC1 in patients with type $2 \mathrm{AIH}$ and evaluate whether a correlation exists between LKM1/LC1 concentra-
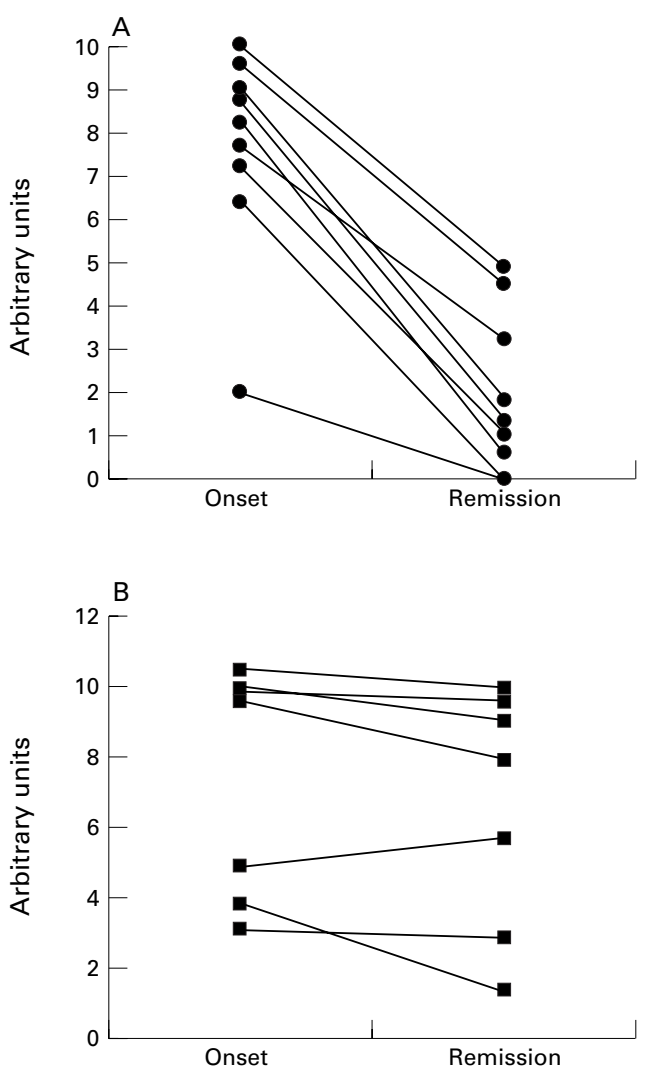

Figure 1 Liver cytosol antibody type 1 (LC1) (A) and liver/kidney microsomal antibody type 1 (LKM1) (B) concentrations were evaluated at onset and during treatment induced remission in patients with type 2 autoimmune hepatitis. LC1, but not LKM1, was decreased significantly by immunosuppressive therapy during which biochemical and immunological parameters were normalised $(F$ test $=87.84 p=0.0001$; factorial analysis of variance). tion and the activity of liver disease, in terms of hepatic necroinflammation, at the time of diagnosis and during treatment induced remission in patients suffering from type $2 \mathrm{AIH}$.

\section{Materials and methods}

PATIENTS

Twenty one patients with cryptogenic liver disease were selected on the basis of LKM1 and/or LC1 positivity. Known viral markers such as hepatitis B surface antigen, antihepatitis $C$ virus, and immunoglobulin $M$ (IgM) anti-hepatitis A virus were all absent. In addition, they were also tested for hepatitis $C$ virus RNA, and were all negative. Genetic causes of liver disease were excluded on the basis of normal serum $\alpha_{1}$-antitrypsin, transferrin, and ceruloplasmin values. Eighteen patients $(86 \%)$ were women, and at onset the median age was 10 years (range 1-30). Presenting symptoms were jaundice (five cases, $24 \%$ ), fatigue (five cases, $24 \%$ ), and prolonged acute hepatitis (three cases, $14 \%$ ), whereas the remaining eight patients $(38 \%)$ were symptomless and were diagnosed after the occasional observation of abnormal liver function tests. At onset, the median serum aspartate aminotransferase value was 9.3 times the upper normal value (range 1-70) and the median serum alanine aminotransferase (ALT) value was 15 times the upper normal value (range 2-80). Polyclonal hypergammaglobulinaemia (median $26 \mathrm{~g} / \mathrm{l}$, range 14-48 g/l) and increased IgG levels (median $31 \mathrm{~g} / \mathrm{l}$, range 19-57 g/l) were also observed. Prothrombin time was altered in 11 patients (median $44 \%$, range $22-67 \%$ ), and total bilirubin was increased in 12 (median 5.6 $\mathrm{mg} / \mathrm{dl}$, range $2-28 \mathrm{mg} / \mathrm{dl}$ ). Hepatomegaly was present in nine cases (43\%), and splenomegaly in ten (48\%). Liver biopsy was performed in 18 of the 21 patients and showed chronic active hepatitis in $12(67 \%)$, multilobular necrosis in one $(5 \%)$ and active cirrhosis in five $(28 \%)$. The application of the International Autoimmune Hepatitis Group scoring system $^{18}$ allowed the diagnosis of "definite" AIH in 16 patients and of "probable" AIH in the remaining five. Four patients also had the following 

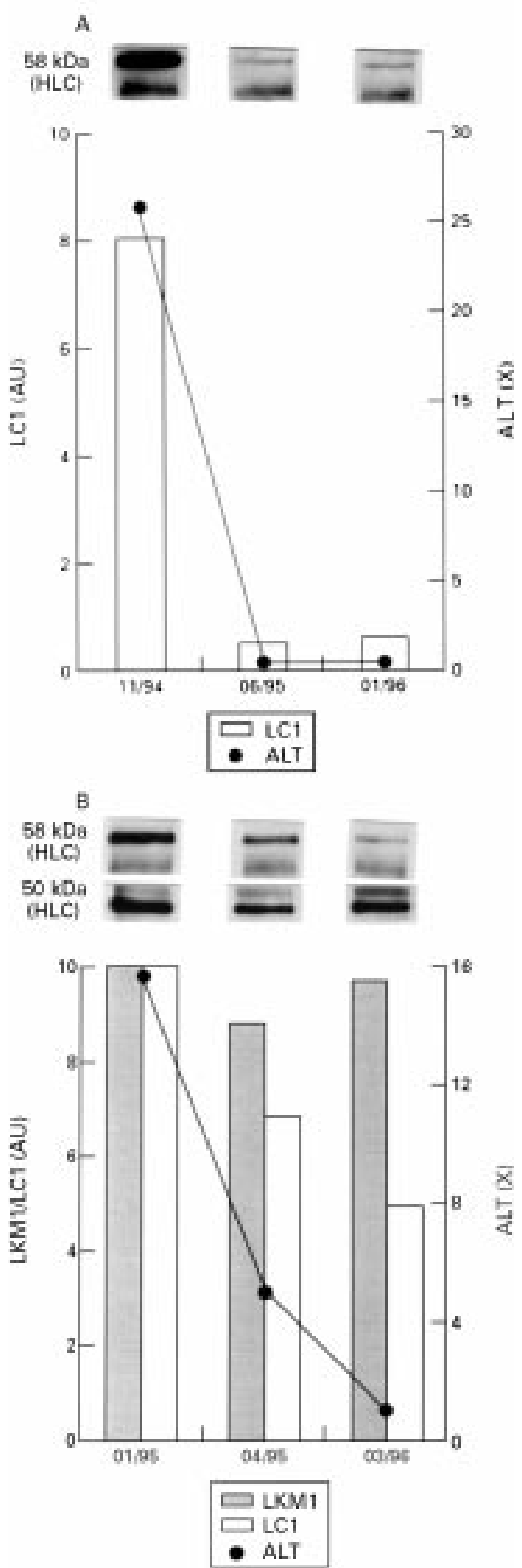

Figure 2 (A) Representative behaviour of liver cytosol antibody type 1 (LC1) concentration and serum alanine aminotransferase (ALT) levels during immunosuppression in patient 19. LC1 immunoreactivity against the $58 \mathrm{kDa}$ (kD) human liver cytosolic (HLC) polypeptide was evaluated by immunoblotting visualised by enhanced chemiluminescence and quantified by densitometric analysis. A clear correlation was observed between LC1 concentration and ALT levels. (B) Independent behaviour of LC1 with respect to liver/kidney microsomal antibody type 1 (LKM1) in patient 13. LKM1 reactivity against the $50 \mathrm{kDa}$ human liver microsomal (HLM) protein - that is, CYP2D6-remained essentially unchanged. An additional $52 \mathrm{kDa}$ band, corresponding to residual human IgG both in microsomal and cytosolic fractions, is also evident. autoimmune disorders: coeliac disease (two), insulin-dependent diabetes mellitus + sicca syndrome (one), hypothyroidism + vitiligo + lichen ruber planus (one). Table 1 gives the main clinical, biochemical, immunological, and histological features for each patient. Eighteen patients $(86 \%)$ were given immunosuppressive therapy (steroids in 11 and steroids + azathioprine in seven), whereas the remaining three patients, all with decompensated liver cirrhosis at the time of diagnosis, received supportive care and were referred to liver transplantation centres. All 18 patients receiving immunosuppressive therapy gradually normalised ALT and $\gamma$-globulin within one to four months. Nine patients, after persistent normalisation of liver function tests, progressively reduced immunosuppressive therapy until withdrawal, but a new hepatitic flare rapidly ensued, which again was controlled by steroid administration.

IMMUNOLOGICAL STUDIES

Serum samples were available for 18 patients at the time of clinical onset or diagnosis, whereas in the remaining three cases sera were only available during follow up. LKM1 and LC1 reactivities were evaluated using different and complementary techniques such as indirect immunofluorescence, counterimmunoelectrophoresis, and immunoblotting visualised by enhanced chemiluminescence. In addition, immunoblotting reactivities were quantified by densitometric analysis.

\section{Indirect immunofluorescence}

Sera diluted 1:10 in phosphate buffered saline were tested on snap frozen sections of rat liver, kidney, and stomach. The second antibody, directed against human immunoglobulin, was conjugated with fluorescein (anti-human polyvalent immunoglobulin IgA, IgG, IgM fluorescein isothiocyanate conjugate; Sigma ImmunoChemicals, St Louis, MO, USA). The immunomorphological patterns of reactivity were assessed under a fluorescence microscopy (Orthoplan; Leitz, Wetzlar, Germany) and classified as LKM1 or LC1 according to the original description. ${ }^{7}$

Counterimmunoelectrophoresis

Undiluted sera were seeded in single wells on agarose plates (agarose $1 \%$ in $0.075 \mathrm{M}$ barbitone buffer, $\mathrm{pH}$ 8.3). After the first electrophoretic run at $16 \mathrm{~mA}$ for 15 minutes, human liver cytosol was added as antigenic source, and a second electrophoretic run at 20 $\mathrm{mA}$ for another 30 minutes was completed. After washing and drying, immunoprecipitin lines were stained with $0.1 \%$ Coomassie blue dye. Sera were considered positive for LC1 only if the precipitin lines gave identity reaction with an LC1 positive reference serum. ${ }^{17}$

Immunoblotting and quantitative densitometric analysis

Human liver microsomal or cytosolic proteins (600 $\mu \mathrm{g}$ per gel) were separated by sodium dodecyl sulphate (SDS)-polyacrylamide gel electrophoresis (PAGE) in 10\% SDS-PAGE 

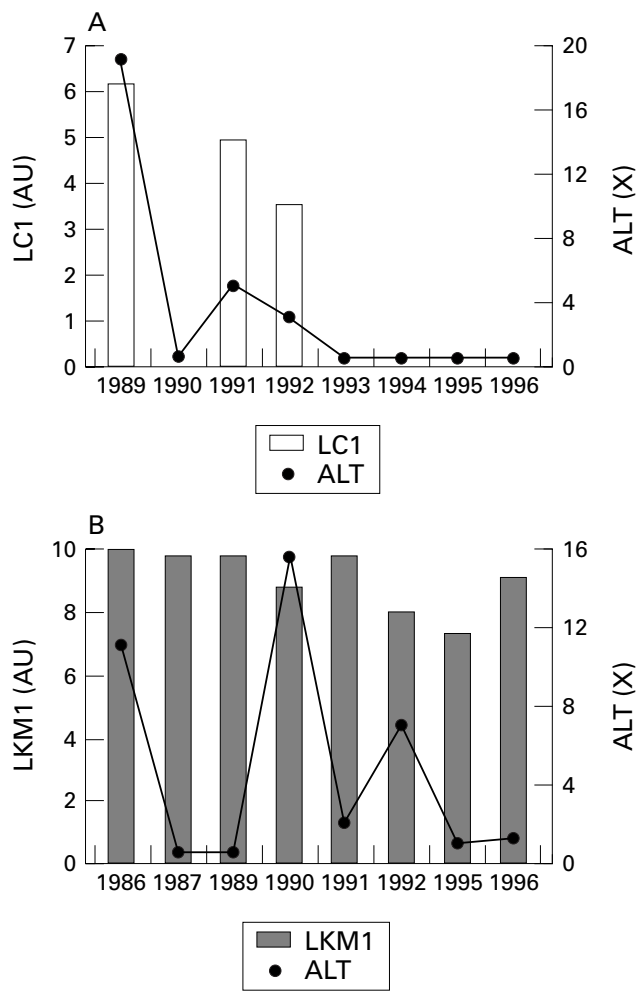

Figure 3 Liver cytosol antibody type 1 (LC1) (A; patient 12) and liver/kidney microsomal antibody type 1 (LKM1) (B; patient 9) concentrations were sequentially reported in parallel with serum alanine aminotransferase (ALT) levels. ALT flares occurred during immunosuppression

withdrawal. LC1 concentration reflects ALT levels at onset, during the first remission, during hepatic flares, and again during the subsequent remission. LKM1 concentration, on the other hand, was consistently elevated throughout follow up, independent of biochemical remission or ALT flares.

minigels (Mini-Protean II System; Bio-Rad Laboratories, Richmond, CA, USA) and transblotted on to nitrocellulose filters, which were then incubated in a blocking solution (Tris buffered saline containing $5 \%$ skimmed milk and $0.1 \%$ Tween 20) for one hour at room temperature. The filters were then cut into strips, and each strip was incubated for two hours at $4^{\circ} \mathrm{C}$ with LKM1 and LC1 positive sera diluted 1:2500, 1:10000 and 1:100 000 in blocking solution. The strips were then washed three times in Tris buffered saline containing $0.1 \%$ Tween 20 , and then incubated for two hours at room temperature in blocking solution with the second antibody diluted 1:100 000 (peroxidase conjugated rabbit anti-human IgG; Dako, Copenhagen, Denmark). After further washing, the colorimetric reaction was developed with an enhanced chemiluminescence kit (Amersham Life Science, Amersham, Bucks, UK) and impressed on $x$ ray films (X-OMAT AR; Eastman Kodak, Rochester, NY, USA). The films were scanned using a GS-670 Imaging Densitometer (Bio-Rad) and analysed with the Molecular Analys/PC software, version 1.1.1 (Bio-Rad). LKM1 positive sera reacted with a $50 \mathrm{kDa}$ microsomal polypeptide, and LC1 positive sera with a 58 $\mathrm{kDa}$ cytosolic polypeptide. Fluctuations of both LKM1 and LC1 levels were best appreciated at a serum dilution of 1:2500, which was used throughout all the subsequent experi- ments. The presence of CYP2D6 in the microsomal preparation was established using the monoclonal antibody BI-114/2, ${ }^{19}$ a gift from Professor U A Meyer (Biozentrum, University of Basel, Switzerland). Samples obtained from 20 healthy blood donors were also tested as negative controls. As a positive control, we used the serum obtained at onset from patient 13, positive for both LKM1 (titre 1/10 240, by indirect immunofluorescence) and LC1 (titre $1 / 256$, by counterimmunoelectrophoresis). The immunoblotting reactivity of such a positive control against the $50 \mathrm{kDa}$ microsomal protein (CYP2D6) and the $58 \mathrm{kDa}$ cytosolic polypeptide was given the value of 10 arbitrary units (AU) for both LKM1 and LC1. A healthy blood donor serum was given the value of 0 AU. Using these two sera as standards for each nitrocellulose filter in all the experiments, a linear regression curve was generated which allowed the estimation, as interpolated values, of LKM1 and LC1 concentration in all the other serum samples.

\section{STATISTICS}

Factorial analysis of variance was used where appropriate.

\section{Results}

Seven patients $(33 \%)$ were positive for isolated LKM1, nine (43\%) for isolated LC1 and five $(24 \%)$ for LKM1 and LC1. By indirect immunofluorescence, 12 patients showed the typical pattern of LKM1, and nine that of LC1. In five LKM1 positive cases, counterimmunoelectrophoresis experiments showed the presence of associated LC1. By counterimmunoelectrophoresis, all 14 LC1 positive cases (nine isolated and five with associated LKM1) gave an identity reaction with the positive reference serum. All 12 patients positive for LKM1 by indirect immunofluorescence reacted in immunoblotting experiments with the $50 \mathrm{kDa}$ microsomal polypeptide - that is, CYP2D6and all the 14 patients positive for LC1 by counterimmunoelectrophoresis recognised the $58 \mathrm{kDa}$ cytosolic polypeptide. At presentation, the median value of LKM1 concentrations, evaluated as the $50 \mathrm{kDa}$ microsomal band, was 9.6 AU (range 3.2-10.5), whereas the median value of $\mathrm{LC} 1$, assessed as the $58 \mathrm{kDa}$ cytosolic band, was 8.2 (range 2-10).

Sequential serum samples from 13 patients (four isolated LKM1, six isolated LC1 and three LKM1+LC1) were studied from onset throughout follow up (median 36 months, range 7-115 months) to quantitatively evaluate LKM1 and LC1 concentration during drug induced remission of the liver disease. A median of three samples for each patient (range 2-10) was obtained, accounting for a global number of 58 serum samples studied. Of the nine LC1 positive patients, all reactive with the $58 \mathrm{kDa}$ cytosolic polypeptide at onset, during remission two lost LC1 completely and in seven LC1 concentration was reduced by more than $50 \%$ ( $F$ test $=87.84, p=0.0001$; fig $1 \mathrm{~A})$. In contrast, of the seven LKM1 positive patients, all reactive with the $50 \mathrm{kDa}$ microsomal polypeptide, during remission six main- 
tained an LKM1 concentration similar to the onset concentration, whereas in only one patient LKM1 was reduced by more than $50 \%$ ( $\mathrm{F}$ test $=3.06, \mathrm{p}=0.10$; fig $1 \mathrm{~B})$.

In indirect immunofluorescence experiments, LKM1 was always detectable before and after remission in six LKM1 positive cases out of seven, whereas four of six isolated LC1 lost the antibody by indirect immunofluorescence, and three of four by counterimmunoelectrophoresis as well.

Figure 2(A) illustrates the typical LC1 response after immunosuppression and ALT normalisation (refers to patient 19), whereas fig 2(B) shows the dissociated behaviour of LC1 and LKM1 in patient 13 at onset and during disease remission.

Interestingly, LC1 concentration seemed to fluctuate with ALT levels, at onset, during disease remission, during reactivation of liver necroinflammation after immunosuppression tapering or withdrawal, and after reinstitution of corticosteroids and control of ALT flares, as illustrated in the case of patient 12 (fig $3 \mathrm{~A}$ ). In contrast, such a strict correlation between autoantibody concentration and liver necrosis was not observed in LKM1 positive patients, as shown by the representative case of patient 9 (fig 3B).

\section{Discussion}

This study describes the behaviour of humoral autoimmune reactions, namely LKM1 and LC1 concentration, in the clinical setting of type $2 \mathrm{AIH}$, before and during immunosuppressive treatment. In our patients the pharmacological treatment, which is mandatory to contain a rapidly progressive liver disease, not only effectively controlled the hepatic damage through generalised immunosuppression which led to ALT reduction and normalisation, but also abated the circulating levels of LC1 resulting in its disappearance, whereas LKM1 concentration was not similarly affected, even in the same patient. Our observations appear to be in contrast with a previous report on four LKM1/LC1 positive patients, in whom, after immunosuppression, LKM1 cleared first, and LC1 later on. ${ }^{20}$ In that report, immunodiffusion and immunofluorescence with immunoabsorption were used to quantify LKM1 and $\mathrm{LC} 1$, and it is difficult to compare different results obtained with different techniques, both considered to be less sensitive than immunoblotting visualised by enhanced chemiluminescence. A possible explanation for such a discrepancy is that immunodiffusion and immunofluorescence are more likely to detect reactivity to conformational epitopes, whereas immunoblotting detects reactivity to "linearised" epitopes. As far as LKM1 is concerned, the availability of CYP2D6 expressed in its correct conformational structure within a eukaryotic system ${ }^{21}$ will clarify whether the humoral autoreactivity against conformational CYP2D6 epitopes differs from that targeting "linearised" CYP2D6 epitopes.

The observation that humoral LC1 autoreactivity correlates strictly with hepatocyte injury suggests that LC1 antigen may be an important liver specific target of the autoimmune attack. By using the sensitive and specific technique of immunoblotting visualised by chemiluminescence and quantified by densitometric analysis, we showed that LC1 concentration, in contrast with LKM1, correlates strongly with ALT levels before and during immunosuppressive treatment in patients with type 2 AIH. In addition, ALT flares following immunosuppression tapering or withdrawal were characterised by an increase in LC 1 concentration, but not in LKM1. The close correlation between LC1 concentration and ALT level during the different phases of the liver disease points to a direct involvement of LC1 autoreactivity in the process of liver targeted autoimmune attack. Although LC1 antigen appears to reside intracellularly-that is, within an immunologically safeguarded site - the possibility cannot be ruled out that, in keeping with other autoantibodies that target cytoplasmic antigens such as anti-CYP2D $6,{ }^{11}$ anti-mitochondrial antibodies, ${ }^{22}$ and antiribosomal P-protein antibodies, ${ }^{23}$ the same or an immunologically related target mimicking the intracellular counterpart may be exposed and accessible on the hepatocyte plasma membrane. The search for a liver specific target accounting for the autoimmune attack in the course of AIH has long been the focus of multiple efforts from various laboratories. Over the years, several immunoreactivities directed against liver antigens have been described, such as liver membrane antigen, ${ }^{24}$ human hepatocyte plasma membrane antigen, ${ }^{25}$ sulphatide, ${ }^{26}$ liver specific membrane lipoprotein, ${ }^{27}$ and its main constituent asialoglycoprotein receptor (ASGP-R). ${ }^{28}$ Today, antigenic preparations such as liver membrane antigen and liver specific membrane lipoprotein are largely of historic interest, antibodies directed against human hepatocyte plasma membrane antigen were studied only in a limited number of cases, and anti-sulphatide antibodies undoubtedly have a low disease specificity. To date, the only liver specific humoral and cellular reactivity that has been investigated in a large number of patients with AIH is that against ASGP-R, although such a reactivity is not restricted to such patients, and a strong correlation has been found between ASGP-R titre and histologically assessed severity of liver disease, but not with biochemical parameters of liver injury. ${ }^{29}$

In addition to its diagnostic significance, the hypothesis can be made that LC1 autoreactivity may represent an additional immunological pathway leading to hepatocyte damage in the course of type $2 \mathrm{AIH}$. To corroborate this hypothesis further, molecular cloning and identification of the LC1 antigen should be pursued in order to elucidate more precisely the nature of this liver specific protein, and efforts should be aimed at demonstrating the presence of LC1 antigen or LC1-like molecules exposed on the outer surface of the hepatocyte plasma membrane.

\footnotetext{
1 Czaja AJ. Autoimmune hepatitis. Evolving concepts and treatment strategies. Dig Dis Sci 1995;40:435-56.

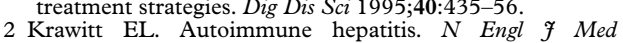
Krawitt EL. Autoi
1996;334:897-903.
} 
3 Maggiore G, Bernard O, Homberg JC, et al. Liver disease associated with anti-liver-kidney microsome antibody in

4 Homberg JC, Abuaf N, Bernard O, et al. Chronic active hepatitis associated with antiliver/kidney microsome antibody type 1: a second type of "autoimmune" hepatitis. Hepatology 1987;7:1333-9.

5 Czaja AJ, Manns MP. The validity and importance of subtypes in autoimmune hepatitis: a point of view. $A m \mathcal{F}$ Gastroenterol 1995;90:1206-11

6 Gregorio GV, Portmann B, Reid F, et al. Autoimmune hepatitis in childhood: a 20-year experience. Hepatology 1997;25:541-7.

7 Martini E, Abuaf N, Cavalli F, et al. Antibody to liver cytosol (anti-LC1) in patients with autoimmune chronic active hepatitis type 2. Hepatology 1988;8:1662-6.

8 Manns MP. Cytoplasmic autoantigens in autoimmune hepatitis: molecular analysis and clinical relevance. Semin Liver Dis 1991;11:205-14.

9 Yamamoto AM, Cresteil D, Boniface O, et al. Identification and analysis of cytochrome P450IID6 antigenic sites and analysis of cytochrome P450IID6 antigenic sites recognized by anti-liver-kidney microsome type-1

10 Muratori L, Lenzi M, Ma Y, et al. Heterogeneity of liver/kidney microsomal antibody type 1 in autoimmune hepatitis and hepatitis C virus related liver disease. Gut 1995;37:406-12.

11 Loeper J, Descatoire V, Maurice M, et al. Cytochromes P-450 in human hepatocyte plasma membrane: recognition by several autoantibodies. Gastroenterology 1993;104: 203-16.

12 Yamamoto AM, Mura C, De Lemos-Chiarandini C, et al. Cytochrome P450IID6 recognized by LKM1 antibody is not exposed on the surface of hepatocytes. Clin Exp Immunol 1993;92:381-90.

13 Vergani D, Mieli-Vergani G. Type II autoimmune hepatitis: the conundrum of cytochrome P450IID6. Clin Exp Immunol 1993;92:367-8.

14 Abuaf N, Johanet C, Chretien P, et al. Characterization of the liver cytosol antigen type 1 reacting with autoantibodies the liver cytosol antigen type 1 reacting with autoantibo
in chronic active hepatitis. Hepatology 1992;16:892-8.

15 Lenzi M, Manotti P, Muratori L, et al. Liver cytosolic 1 antigen-antibody system in type 2 autoimmune hepatitis and hepatitis C virus infection. Gut 1995;36:749-54.

16 Han S, Tredger M, Gregorio GV, et al. Anti-liver cytosolic antigen type 1 (LC1) antibodies in childhood autoimmune liver disease. Hepatology 1995;21:58-62.

17 Muratori L, Cataleta M, Muratori P, et al. Detection of antiliver cytosol antibody type 1 (anti-LC1) by immunodiffusion, counterimmunoelectrophoresis and immunoblotting. comparison of different techniques. 7 Immunol Methods 1995;187:259-64.
18 Johnson PJ, McFarlane IG. Meeting report: International Autoimmune Hepatitis Group. Hepatology 1993;18:9981005.

19 Zanger UM, Hauri HP, Loeper J, et al. Antibodies against human cytochrome P450-db1 in autoimmune hepatitis type II. Proc Natl Acad Sci USA 1988;85:8256-60.

20 Abuaf N, Johanet C, Soulie E, et al. Anti-liver cytosol antibodies in hepatology: autoimmune hepatitis, viral hepatitis $\mathrm{C}$ and graft-versus-host disease. In: Meyer zum Büschenfelde KH, Hoofnagle JH, Manns M, eds. Immunology and liver Falk Symposium 70. London: Kluwer ogy and liver Falk Symposium 70.

21 Ma Y, Gregorio G, Gäken J, et al. Establishment of a novel radioligand assay using eukaryotically expressed cytochrome P4502D6 for the measurement of liver kidney microsomal type 1 antibody in patients with autoimmune hepatitis and hepatitis C virus infection. 7 Hepatol 1997;26: 1396-402.

22 Joplin R, Gordon Lindsay J, Johnson GD, et al. Membrane dihydrolipoamide acetyltransferase (E2) on human biliary epithelial cells in primary biliary cirrhosis. Lancet 1992; 339:93-4.

23 Koren E, Wolfson A, Koscec M, et al. Autoantibodies to ribosomal $\mathrm{P}$ proteins react with a plasma membranerelated target on human cells. F Clin Invest 1992;89:123641.

24 Hopf U, Meyer zum Büschenfelde KH, Arnold W. Detection of a liver membrane autoantibody in HBsAgnegative chronic active hepatitis. $N$ Engl f Med 1976;294: 578-82.

25 Swanson NR, Reed WD, Yarred LJ, et al. Autoantibodies to isolated human hepatocyte plasma membranes in chronic active hepatitis. II. Specificity of antibodies. Hepatology 1990;11:613-21.

26 Toda G, Ikeda Y, Kashigawi M, et al. Hepatocyte plasma membrane glycosphingolipid reactive with sera from patients with autoimmune chronic active hepatitis: its identification as sulfatide. Hepatology 1990;12:664-70.

27 Meyer zum Büschenfelde KH, Miescher PA. Liver-specific antigens, purification and characterization. Clin Exp Immunol 1972;10:89-102.

28 McFarlane IG, McFarlane BM, Major GN, et al. Identification of the hepatic asialoglycoprotein receptor (hepatic lectin) as a component of liver-specific membrane lipoprotein (LSP). Clin Exp Immunol 1984;55:347-54.

$29 \mathrm{McF}$ arlane BM. Hepatocellular membrane antigens. In: McFarlane IG, Williams R, eds. Medical Intelligence Unit. Molecular basis of autoimmune hepatitis. Austin: RG Landes Company, 1996:75-104. 